\title{
Masking the Discolored Enamel Surface with Opaquers before Direct Composite Veneering
}

\author{
Spaveras Andreas ${ }^{1}$, Vjero Osela ${ }^{2}$, Anagnostou Maria ${ }^{3}$ and Antoniadou Maria ${ }^{3 *}$ \\ ${ }^{1}$ Department of Operative Dentistry, Dental School, National and Kapodistrian University of Athens, Dentist, Greece \\ ${ }^{2}$ Department of Operative Dentistry, Dental School, National and Kapodistrian University of Athens, Postgraduate Student, Greece \\ ${ }^{3}$ Department of Operative Dentistry, Dental School, National and Kapodistrian University of Athens, Lecturer, Greece
}

Received: June 08, 2015; Accepted: July 09, 2015; Published: July 15, 2015

*Corresponding author: Antoniadou Maria, Department of Operative Dentistry, Dental School, National and Kapodistrian University of Athens, Lecturer, Greece, E-Mail: mantonia@dent.uoa.gr

\begin{abstract}
Conservative and aesthetic treatment of a single discolored tooth in the anterior zone is a restorative challenge. Intra-pulpal hemorrhage, dental trauma and endodontic treatment are common reasons for tooth discoloration. If a bleaching procedure cannot be implemented or has been tried without pleasing results, other conservative treatment plans should be considered under the scope of minimal intervention. A relevant application would be the construction of a direct composite veneer. The problem is that composite resins have inherent limitations in their opacification ability, especially when increments are very thin. Thus, in order to match the chromaticity of the adjacent teeth, the use of opaquers is suggested. Opaquers are highly pigmented resinous materials, containing metal oxides that are responsible for their potent opacification ability, characteristic tint and saturation. These materials can effectively mask a dark enamel surface, after minimal tooth preparation. Different composite layers can be stratified on the masked substrate according to the layering technique, emulating the natural dentine and enamel tissues. The aim of this study is to describe the use of opaquers in masking discolored enamel surfaces, prior to direct veneering with resin composites.
\end{abstract}

Keywords: Resin composite veneers; Layering technique; Opaquers; Anterior aesthetic zone; Tooth discoloration

\section{Introduction}

The rapid evolution of adhesive dentistry has established composite resins as one of the most commonly used restorative materials in daily practice. Its advanced mechanical properties and ability of emulating the chromatic and textural characteristics of dental tissues in a direct and cost saving manner, has driven the anterior dental treatment to a more aesthetic yet minimallyinvasive approach. Anterior aesthetics can gravely influence the self-esteem and psychosocial profile of the patient; therefore, they must be confronted with equal importance as any other functional step during treatment planning.

The fundamental principle of any anterior esthetic restoration is the perceived harmony with the adjacent soft and hard dental tissues. Achieving aesthetic composite restorations in the anterior region demands not only skilled hands and training, but a deeper understanding of the chromatic characteristics of teeth, as well as optical phenomena such as fluorescence, opalescence, counter-opalescence, translucency and opacity [1].

Emulating the natural dentition with composite resin can be an even greater clinical challenge in the case of a single discolored anterior tooth. Discoloration can be caused by a variety of reasons, including intra-pulpal hemorrhage, dental trauma and endodontic treatment [2]. Masking the discolored enamel surface with opaquers and tints before direct composite veneering may be a necessary step, especially when it comes to minimal tooth preparation.

The purpose of this article is the description of a minimally invasive direct approach to an anterior single tooth discoloration with the aid of opaquers. Clinical tips related to the application of opaquers as well as common mistakes that can hamper the final appearance of the restoration are presented.

\section{Causes of Tooth Discoloration}

The differential diagnosis of tooth discoloration is a fundamental step for the selection of the correct treatment plan. There are two types of tooth discolorations for vital teeth. Those caused by intrinsic and those caused by extrinsic factors (Table 1).

Intrinsic discolorations occur following a change in the structural composition or thickness of the dental hard tissues during odontogenesis, resulting in alteration of the light transmitting properties of teeth [2]. The intrinsic discoloration of a single anterior tooth is most often related to dental trauma, due to the rupture of the blood vessels in the pulp chamber. Discoloration of more than one tooth can be caused by genetic disorders such as dentinogenesis or amelogenesis imperfecta, where concerning the latter one, the color change of the affected teeth ranges from white opaque to yellow depending on its subtype [2]. Furthermore, fluorosis can cause white opaque or brown stains while certain tetracyclines are responsible for yellow to brown and grey discolorations of the affected teeth. Minocycline hydrochloride for example, a drug used mainly for 
acne treatment, is a tetracycline derivative, capable of pigmenting teeth in only a week of intake [2].

Additionally, in endodontically treated teeth various reasons are responsible for the commonly seen discolorations (Figure 1A,B). Incomplete removal of the coronal pulp during endodontic treatment, intra-pulpal hemorrhage, dentine sclerosis, certain restorative and endodontic materials such as sealers, medicaments or pastes, are all linked to non-vital tooth discoloration [3]. Finally, changes in the water content after endodontic treatment is reported to alter the optical properties of non-vital teeth too [4].

Extrinsic discolorations on the other part can be caused by pigments or by compounds that chemically interact with the dental surface, causing its staining. Its severity is influenced among others by the salivary flow and composition, as well as patient dietary habits. Tannin, a biomolecules contained in beverages and food, as well as the use of tobacco can lead to brown or black discolorations, respectively. Chlorexidine mouth rinses or the accumulation of metallic compounds such as iron, manganese and mercury are only some contributors to the multifactorial etiology of extrinsic tooth discoloration [2].

Restorative procedures such as veneering are proposed mainly when dealing with intrinsic discolorations of various degrees, while extrinsic ones can be faced with preventive oral hygiene measures or bleaching.

\section{Opaque Composite Resins}

Enamel is a translucent tissue comprised of mineralized

Table 1: Common extrinsic and intrinsic causes of tooth discoloration.

\begin{tabular}{|l|l|}
\hline Extrinsic & Intrinsic \\
\hline $\begin{array}{l}\text { Food and drinks } \\
\text { ( ex. Tea, coffee, wine, colas) }\end{array}$ & Pulpal necrosis \\
\hline $\begin{array}{l}\text { Tobacco use } \\
\text { ( ex. Chewing or smoking } \\
\text { tobacco) }\end{array}$ & $\begin{array}{l}\text { Calcification of the pulp chamber } \\
\text { (ex. Dental trauma) }\end{array}$ \\
\hline $\begin{array}{l}\text { Bad oral hygiene } \\
\text { (ex. Plaque deposition) }\end{array}$ & $\begin{array}{l}\text { Dental materials } \\
\text { (ex. Extensive amalgam restorations) }\end{array}$ \\
\hline $\begin{array}{l}\text { Topical medications } \\
\text { ( ex. Chlorexidine mouthrinses) }\end{array}$ & $\begin{array}{l}\text { Familial/congenital disorders } \\
\text { (ex. Amelogenesis imperfect) }\end{array}$ \\
\hline $\begin{array}{l}\text { Metallic compounds } \\
\text { (ex. Accumulation of iron, } \\
\text { manganese, mercury) }\end{array}$ & $\begin{array}{l}\text { Metabolic disorders } \\
\text { (ex. Alkaptonuria) }\end{array}$ \\
\hline $\begin{array}{l}\text { Enamel wear } \\
\text { (ex. Abrasion, attrition, erosion) }\end{array}$ & $\begin{array}{l}\text { Antibiotic drugs } \\
\text { (ex. Minocycline, tetracycline) }\end{array}$ \\
\hline & $\begin{array}{l}\text { Dental Fluorosis } \\
\text { (ex. Excessive fluoride uptake) }\end{array}$ \\
\hline
\end{tabular}

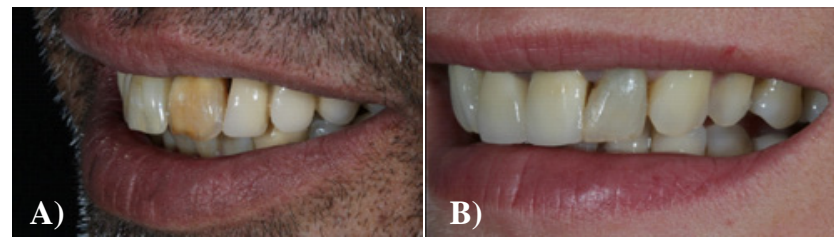

Figure 1: Discolored teeth A) Left central incisor of a 55 years old man B) Left lateral incisor in 45 years old woman. prisms that reflect, transmit and absorb different wavelengths of light, ranging from 400 to $700 \mathrm{~nm}$. When a visible ray penetrates the enamel layer, a certain part reaches the dentine and is ultimately reflected towards the surface. This phenomenon results in the dentine's and enamel's color estimation. The cervical enamel has a thickness of $0,3 \mathrm{~mm}$. Therefore it is easier for the clinician to determine the dentine's color and consequently the tooth's overall shade from this region. In the mid third of the tooth, the enamel thickness ranges between 0,8-1 $\mathrm{mm}$, whereas in the incisal third, the enamel reaches its maximum thickness of 0,9-1,2 $\mathrm{mm}$ [5]. Different translucencies and thicknesses of the dental tissues directly influence the color of the teeth as a combined optical phenomenon of the visible light [6].

Restorative materials that naturally mimic the dental substrates should ideally have the same optical properties as them. The inherent translucency of composite resins, contributes to the imitation of these optical properties [7]. On the other hand, it shows a distinctive difficulty in masking the dark background of a discolored tooth, especially when the tooth's preparation is minimal $[8,9]$. The background reflectivity of the discolored tooth, as well as the inappropriate thickness of the restorative material used, both negatively influence the natural appearance of the restoration [10].

Some resin composite kits offer shades of opaque composite resins. It is reported that translucency in those opaque shades is lower than in dentin or enamel shades [10]. Furthermore, it has been proved that an opaque composite resin with a thickness of $0.5-1.0 \mathrm{~mm}$ was required to mask a C4 shade background color while an opaque layer of 1.0-2.0 mm was required for masking the black background color [8]. Accordingly it was reported elsewhere that the thicknesses of opaque-shade composite resins that masked the $\mathrm{C} 4$ shade and black backings had a thickness of 0.80-1.45 mm and 1.85-2.00 mm, respectively [9]. Thus it can be concluded that for the effective cover of a really discolored tooth substrate, an opaque layer of $0.80-1.45 \mathrm{~mm}$ of thickness is needed. It can be noted though that a palatally inclined discolored tooth may provide this space without the need of sound tooth removal.

Opaquers are highly pigmented resinous materials, containing metal oxides that are responsible for their potent opacification ability, characteristic tint and saturation [10] (Table 2). The ability to mask the background effect through the complex interaction between absorption and scattering can be achieved by adding opacifiers to composite resin, such as titanium oxide or aluminum oxide, thus increasing the light reflected towards the observer [9].

Opaquers are available in a flowable viscosity (e.g. Creative Color, Pink Opaque, Cosmedent, Chicago, IL, USA), in a base-catalyst paste form (e.g. Opaquer dual cured, Bisco, Inc, Schaumburg, IL, USA) or in a powder/liquid one (e.g. Sinfony, Opaquer, 3M ESPE AG, Seefeld, Germany), (Figure 2A,B). Some laboratory opaquers can also be used clinically for masking, while repairing the underlying metal framework of resin veneered crowns or metal framework of chipped porcelain and acrylic fused to metal restorations. The ability to mask a discolored 


\begin{tabular}{|c|c|c|}
\hline Material & Company & Chemical Composition \\
\hline \multirow{2}{*}{$\begin{array}{l}\text { Kolor + Plus, } \\
\text { Opaquer }\end{array}$} & \multirow{2}{*}{$\begin{array}{l}\text { Kerr Corporation, } \\
\text { Orange, CA, USA }\end{array}$} & - Bis-GMA: (80-95 wt. \%), - Inert mineral fillers \\
\hline & & -Catalysts, stabilizers, and pigments \\
\hline \multirow{5}{*}{$\begin{array}{l}\text { Inspiro, } \\
\text { Opaque }\end{array}$} & \multirow{5}{*}{$\begin{array}{l}\text { Edelweiss DR AG, Mercandor, } \\
\text { Switzerland }\end{array}$} & -Bis-GMA, -Barium glass, zinc-Fluoride, nanoparticles: (65.5 wt. \%) \\
\hline & & Particle size: $0.02-3 \mu \mathrm{m}$, Mean particle size: $0.7 \mu \mathrm{m}$ \\
\hline & & Flexural modulus: $6 \mathrm{GPa}$, Flexural strength: $120 \mathrm{MPa}$ \\
\hline & & Compressive strength: $350 \mathrm{MPa}$ \\
\hline & & -Catalysts, stabilizers, and pigments \\
\hline \multirow{2}{*}{$\begin{array}{l}\text { Creative Color, } \\
\text { Pink Opaque }\end{array}$} & \multirow{2}{*}{$\begin{array}{l}\text { Cosmedent, } \\
\text { Chicago, IL, USA }\end{array}$} & Inorganic fillers (10-12\% wt.) \\
\hline & & -Catalysts, stabilizers, and pigments \\
\hline \multirow{11}{*}{$\begin{array}{l}\text { Sinfony, } \\
\text { Opaquer }\end{array}$} & \multirow{11}{*}{$\begin{array}{l}\text { 3M ESPE AG, } \\
\text { Seefeld, Germany }\end{array}$} & Powder ingredients \\
\hline & & - Silane treated quartz (40-50wt. \%), - Calcium fluoride (15-30 wt. \%) \\
\hline & & -Titanium dioxide (15-30 wt. \%), -Lauroyl peroxide (1-5 wt. \%) \\
\hline & & -Isobutylmalonyl-n,n'-dicyclohexylsulfamide (1-5 wt. \%) \\
\hline & & -Silane treated silica (<2 wt. \%), -C.i. pigment yellow $42(<1.5$ wt. \%) \\
\hline & & -Iron hydroxide oxide (<1.5 wt. \%) \\
\hline & & Liquid ingredients \\
\hline & & -Dicyclopentyldimethylene diacrylate (35-45 wt. \%) \\
\hline & & -Methyl methacrylate (30-40 wt. \%), -Monoacrylateacetate (10-20 wt. \%) \\
\hline & & -Vinyl acetate polymer (5-10 wt. \%), -Phosphine oxide (1-5 wt. \%) \\
\hline & & -N,n -dibutylphenylethylamine hydrochloride (<2 wt. \%) \\
\hline \multirow{5}{*}{$\begin{array}{l}\text { Clearfil ST } \\
\text { Opaquer }\end{array}$} & \multirow{5}{*}{ Kuraray America, Inc. } & -Bisphenol A diglycidylmethacrylate (5-25 wt. \%) \\
\hline & & -Triethylene glycol dimethacrylate (<10 wt. \%) \\
\hline & & -Silanated silica filler, -Colloidal silica, -Silanated colloidal silica \\
\hline & & -Dl-Camphorquinone \\
\hline & & -Catalysts, stabilizers, and pigments \\
\hline \multirow{5}{*}{ Monopaque } & \multirow{5}{*}{$\begin{array}{l}\text { Ivoclar Vivadent, } \\
\text { Schaan, Liechtenstein }\end{array}$} & -Bis-GMA, urethane dimethacrylate and triethylene glycol dimethacrylate (54,2 wt. \%) \\
\hline & & -Barium glass, Ytterbium trifluoride, Ba-Al-fluorosilicate glass, spheroid mixed oxide ( 43,1 wt. \%) \\
\hline & & - Total content of inorganic fillers $(23,2 \mathrm{vol} \%)$ \\
\hline & & - Particle size: 0,04-3,0 $\mu \mathrm{m}$, -Mean particle size: $0,7 \mu \mathrm{m}$. \\
\hline & & Catalysts, stabilizers, and pigments (2,7 wt. \%) \\
\hline \multirow{6}{*}{$\begin{array}{l}\text { IPS Empress } \\
\text { Direct Opaque }\end{array}$} & \multirow{6}{*}{$\begin{array}{l}\text { Ivoclar Vivadent, } \\
\text { Schaan, Liechtenstein }\end{array}$} & -Dimethacrylates $(53,9$ wt. \%) \\
\hline & & -Barium glass, ytterbium trifluoride, Ba \\
\hline & & -Alfluorosilicate glass, spheroid mixed oxide (43,4 wt. \%) \\
\hline & & -Total content of inorganic fillers $(23,2 \mathrm{vol} \%)$ \\
\hline & & -Particle size $0,04-3,0 \mu \mathrm{m}$, -mean particle size: $0,7 \mu \mathrm{m}$. \\
\hline & & Catalysts, stabilizers, and pigments (2,7 wt. \%) \\
\hline \multirow{4}{*}{ Cimara Opaque } & \multirow{4}{*}{ Voco, Cuxhaven, Germany } & -Bis-GMA, (10-25 wt. \%), - urethane dimethacrylate (10-25 wt. \%) \\
\hline & & - HEDMA (10-25 wt. \%) \\
\hline & & Catalysts, stabilizers, and pigments (2.5\% wt. \%) \\
\hline & & Benzotriazole derivates $(2.5 \mathrm{wt} . \%)$ \\
\hline \multirow{4}{*}{$\begin{array}{l}\text { OPAQUER Dual } \\
\text { Cured (Base/ } \\
\text { Catalyst) base } \\
\text { - katalytics }\end{array}$} & \multirow{4}{*}{ Bisco, Inc, Schaumburg, IL, USA } & -Bis-GMA. (<40 wt. \%), - Urethane dimethacrylate (<30 wt. \%) \\
\hline & & \{Base ingredient\}, - Triethyleneglycol dimethacrylate (<40 wt. \%) \\
\hline & & - Benzoyl Peroxide $(<2$ wt. \%) \{Catalyst ingredient $\}$ \\
\hline & & Inorganic Fillers: - Glass Filler $<40$ wt. \%, - Amorphous Silica $<20$ wt. \% \\
\hline
\end{tabular}

background or in other words the opacification potential of these laboratory opaquers has been stated as stronger than the clinical ones [11]. Despite their form however, all opaquers should be applied in very thin layers $(0,1-0,5 \mathrm{~mm})[9]$.

The tone of the selected opaquer should be close to the desired shade of the final direct veneer [9]. It is suggested that clinicians should get familiarized with different opaquers in laboratory conditions before trying their clinical application. This would benefit their knowledge concerning opaquers' masking ability, different consistency, handling, possible application errors, optical characteristics and number of layers needed. (Figure 3A,B \& Figure 4).

Color tints on the other hand, are highly pigmented resins that contain metal oxides, which selectively reflect and absorb 


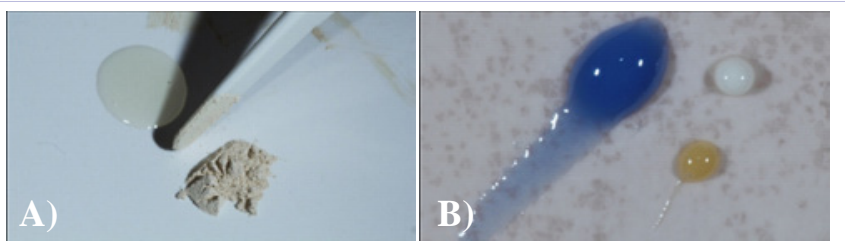

Figure 2: A) Powder-liquid opaquer (Sinfony Opaquer) B) Tints (Chroma and Ice Effect Shades, Inspiro) and color modifier (Blue, Venus).
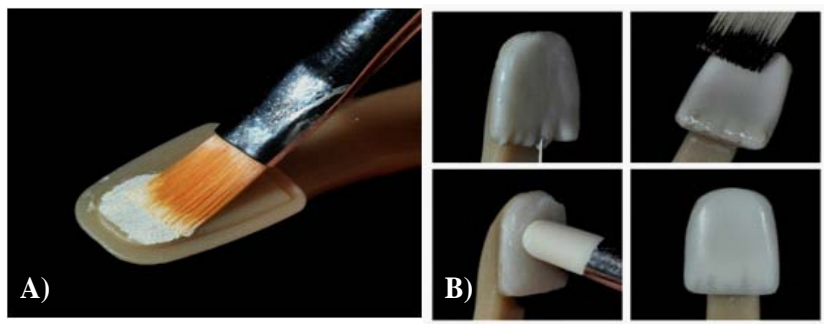

Figure 3: A) Uniform application of the opaquer with a flat painter's brush B) Layering of the plastic analogue with dentin and enamel shades.

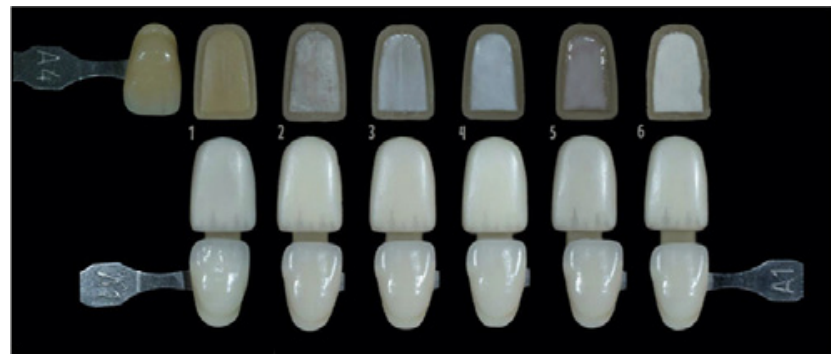

Figure 4: Different opacification results using different opaquers/combinations 1) Without Opaquer, 2) Sinfony (3M) + Ice Effect Shade (Edelweiss), 3) A1 Opaquer (Kerr), 4) Opaque (Edelweiss), 5) Pink Opaquer (Cosmedent), 6) Sinfony (3M).

parts of visible light. This fact is responsible for the distinctive vibrant colors and relative translucency of the tints [12]. Tints can be mixed with opaquers or be applied over them in order to reduce the opacity of the so called "strong" opaquers. Different tint shades can be applied to specific tooth areas with better results. For example, yellow and light brown tints are usually applied on the mid third of the tooth while blue and violet on the mesial, distal and incisal parts.

\section{Clinical Tips for Application of Opaquers}

The application of opaquers on the discolored tooth surface is a crucial step towards the clinical procedure. The clinical tips that follow can secure a more predictable final result.

Selection of the suitable opaquer: Different products show different opacification abilities and while some are able to effectively mask a dark background with only $0,1 \mathrm{~mm}$ thick layer, others may require two or more layers [9]. In heavily discolored dentition, strong opaquers can offer better results and as previously mentioned, the strongest opaquers are those of the powder/ liquid systems. If an opaquer with higher than the needed opacification ability or thickness is applied, the surface may acquire an undesired dull and "lifeless" appearance. Therefore, in cases where a "medium" opacity is needed, it has been suggested to dilute strong opaquers with bis-GMA or fluid resin $[10,12]$. On the other hand, if the amount of the opaquer is not sufficient, the final restoration will have an unfavourable grey tone.

Consistent layering of the opaquer: Unequal layering of the opaquer on the discolored tooth surface is a common clinical mistake [9]. The result of a non homogeneous opaquer layer would be a "spotted" surface. The use of a fine painter's brush coated with minimum quantity of the material or liquid resin can be a useful tool in handling the consistent spread of the opacious modifiers.

Ensuring adequate space for the opaquer: A minimal thickness of $0,4 \mathrm{~mm}$ should be available for the composite stratification after opaquing the discolored substrate [10]. The use of silicone indexes fabricated indirectly in the laboratory or directly in the patient's mouth, can ensure that enough space for composite layering is provided, without unnecessary sound tooth preaparation. If the opaquer is stratified with inadequate amounts of dentine and enamel layers, especially in the cervical region where the enamel is thinner, the veneer will appear unnatural [12].

Generally, the analysis of the chromatic and volumetric characteristics of the natural dentition is crucial to the dentist's understanding. He should nonetheless be able to reproduce them clinically in order to achieve aesthetic results. If the dental discoloration is very strong or if the tooth is more buccally positioned than the adjacent teeth, the tooth tissues should be reduced more in order to provide sufficient space for the masking and layering techniques [10]. Finally, proper finishing and polishing of the composite resin is of utmost importance in cases of direct veneer fabrication.

\section{Presentation of Cases}

\section{Case 1}

A 35-year-old female with a discolored maxillary right central incisor and a malpositioned right lateral incisor (Figure 5A,B), arrived with the desire to improve her smile (Figure 6A,B). The patient reported dental trauma as the cause of the discoloration which had occured almost 15 years ago. According to the acquired dental history of the patient, an external bleaching procedure had been performed almost a decade ago with custom-made trays, but the shade rebound lead to a gradual unpleasant outcome. Clinical examination confirmed the vitality of the right central
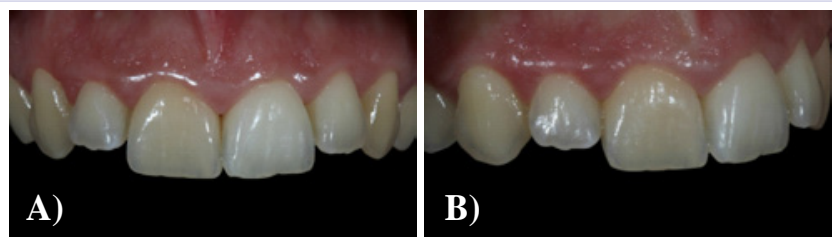

Figure 5: Right lateral and central incisor A) Front view B) Lateral view. 
incisor. An external in-office bleaching procedure was initially suggested to the patient, following the philosophy of minimal tooth intervention. The patient refused to follow any rebleaching treatment. She was determined to have a direct conservative esthetic solution for her upper front teeth. Therefore, the final treatment plan included the direct veneering with composite resins of the central and the reshaping of the lateral incisors.

Tooth shade selection was performed before field isolation to avoid color mismatches due to teeth' dehydration (Figure 7A,B). The right central incisor had a disto-palatal inclination of $0,6 \mathrm{~mm}$ (Figure 8A). A direct diagnostic resin mock-up was transferred to the right central and lateral incisors from a diagnostic waxup (Figure 8B). The patient approved it without any corrections. The tooth needed to be prepared only mesially $(0,3 \mathrm{~mm})$, using a cylindrical round-ended diamond rotary cutting instrument (856, 0,16mm, 10mm, Comet GEBR. BRASSELER) at different angles following the convexity of the tooth, under constant water irrigation. The preparation was within the enamel without sharp line angles for maximum conservation of structures and strength of the resin veneer (Figure 9).

The six upper front teeth were isolated with a medium weight rubber dam (Nictone, MDC Dental, Mexico) and metal clamps (1 Satin Steel, Hu-Friedy Mfg. Co.) were positioned on the premolars over the rubber dam stabilizing it. A 37, $5 \%$ phosphoric acid (Gel-Etchant, Kerr Corporation, Orange, CA, USA) was applied to the prepared enamel for 30 seconds, rinsed with air-water spray
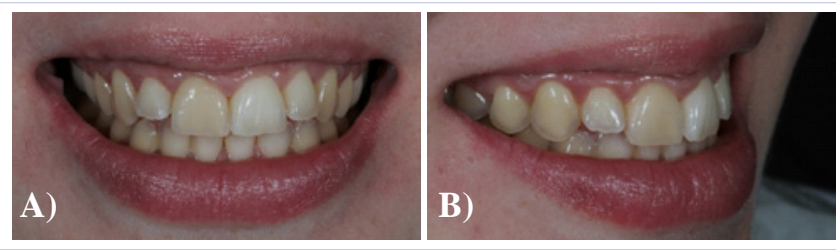

Figure 6: Patient's smile A) Front view B) Lateral view.
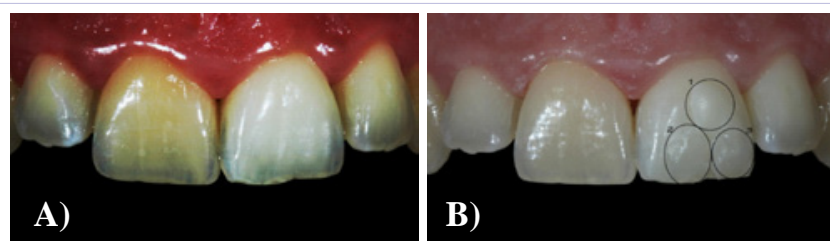

Figure 7: A) Digital processed contrast and brightness in order to highlight the chromatic characteristics of the right central incisor B) Direct intra-oral selection of composite shades to be used for the veneer (1: Enamel A1, 2: Body A2, 3:Body A1).

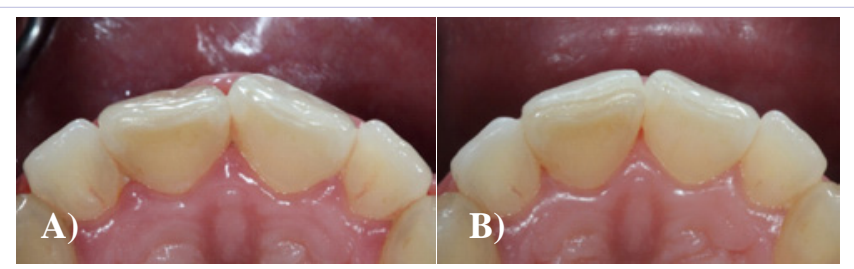

Figure 8: Palatal view of both left central and lateral insisors A) Initial view B) View after direct diagnostic mock up.

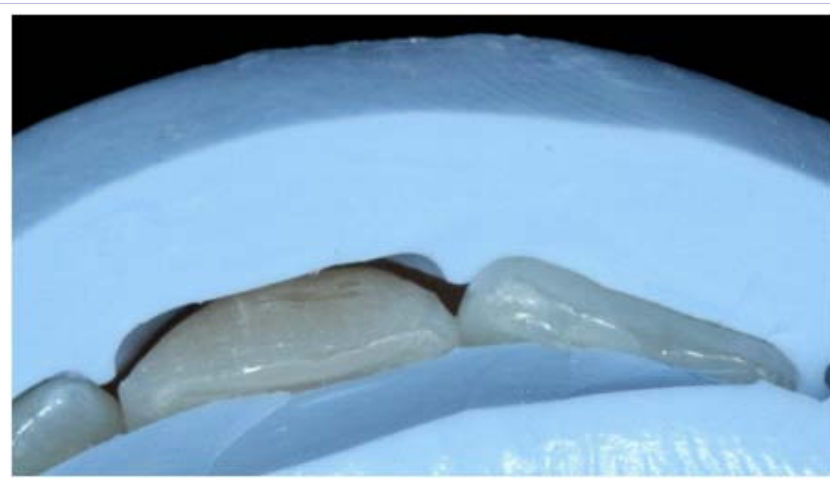

Figure 9: Evaluation of the depth of the preparation with a silicon key.

for the same amount of time and air-dried. Adhesive procedures were performed with a three-step etch-and-rinse system (Optibond FL, Kerr Corporation, Orange, CA, USA), according to the manufacturer's directions of use.

A portion of resinous color modifier (Ice Effect Inspiro, Edelweiss DR AG, Mercandor, Switzerland) was placed (Figure $10 \mathrm{~A}$ ) and spread uniformly in two very thin layers on the discolored enamel surface with a flat painter's brush (Da Vinci, Series 374, Flat No. 4) (Figure 10B). The color modifier was used mainly in order to simulate fluorosis or demineralization surface characteristics. Its selection was based on its ideal flowable consistency, ease of layering but also due to the mild severity of the tooth discoloration which suggested also mild coverage. Each layer was photopolymerized separately with a LED curing device (TechnoGaz, Parma, Italy) for 40 seconds according to manufacturers' directions of use.

A 0,5 mm increment dentin shade (body A2), (Filtek Supreme Ultra, 3M ESPE AG) was stratified and the dentine lobes were sculpted with composite contouring instruments (LM-Arte Set, LM Dental AB, Parainen, Finland), (Figure 11A). Small portion of translucent shade and blue and white tints (Venus, Heraeus Kulzer, Hanau, Germany) were applied in the incisal third. In sequence a thin portion of dentin shade (body A2) was spread over the tints and along the incisal edge in order to reproduce the "halo effect" (Figure 11B). Furthermore color modifiers (Ice and Chroma Effect Shades, Inspiro, Edelweiss DR AG, Mercandor, Switzerland) were also placed incisally and light-cured for 20 seconds in order to emulate the chromatic characterizations of the adjacent central incisor. The applied combination of opaquers was mainly selected in order to increase the chroma value in the incisal third and finally mimic the smooth whitish imperfections of the incisal edge of the adjacent tooth. The reproduction of the enamel layer was recreated with a $0,3 \mathrm{~mm}$ layer of enamel shade (A1 Enamel) (Figure12).

Extra-fine diamond burs and aluminium oxide disks (Sof-Lex, 3M ESPE AG, Seefeld, Germany) were used for finishing and a two-step silicon-rubber polishing cups (FlexiCups, Cosmedent, Chicago, IL, USA) for polishing. Finally, a $5 \mu \mathrm{m}$ diamond polishing paste (Diamond Polish Mint, Ultradent Products Inc, South Jordan) was used, in order to achieve a higher surface gloss. 
Reshaping of the right lateral incisor was completed during the same dental appointment. Restorations (Figure 13A, B) and the overall smile (Figure 14A,B) were finally checked at oneweek recall appointment.

\section{Case 2}

A 37-year-old female with a discolored upper left lateral incisor (Figure 15A,B), was presented at the Postgraduate Clinic of the Department of Operative Dentistry, University of Athens, Greece. The desire was to improve her smile (Figure 16A,B). The endodontic treatment of the left central incisor, performed 2 years ago after root apecification, was evaluated and confirmed as clinically acceptable (Figure 17). The treatment plan then of the discolored left lateral incisor included only the conservative approach of direct veneering -due to its previous heavily processed condition- with the use of a nanocomposite system
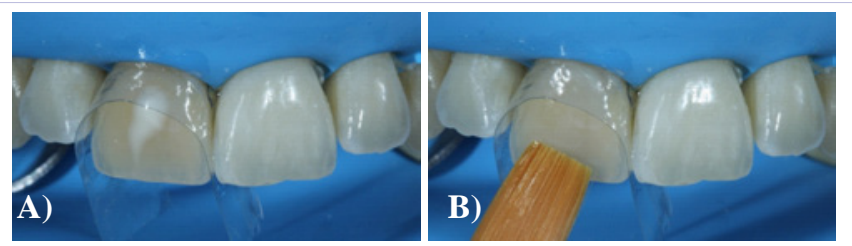

Figure 10: A) Application of a small amount of opaque (Ice Effect Shade) with the tip of the brush B) Uniform layering of the opaque material with a thin painter's brush.
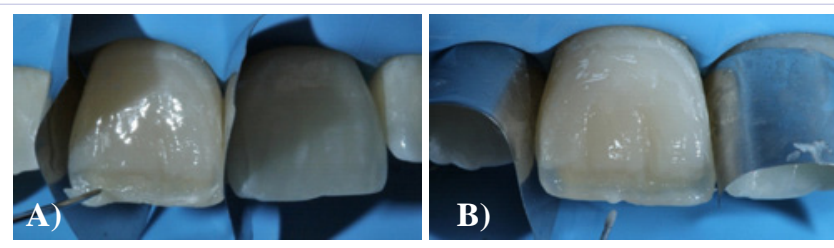

Figure 11: A) Layering of the dentine shade B) Reproduction of the "halo effect" with application of a white color modifier at the incisal edge.

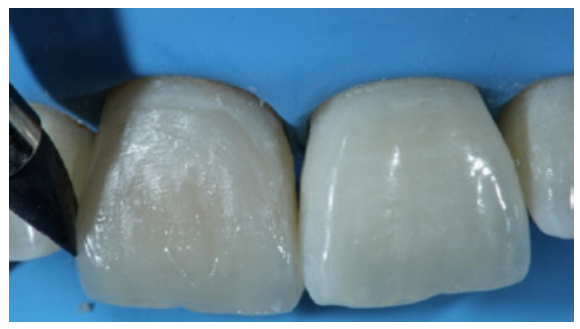

Figure 12: Final layering and contouring of the enamel shade.
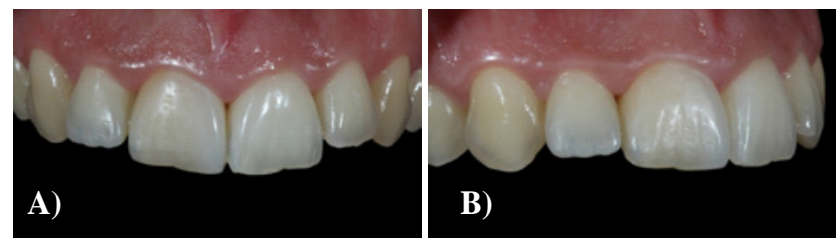

Figure 13: The restored right lateral and central incisors after one week A) Front view B) Lateral view.
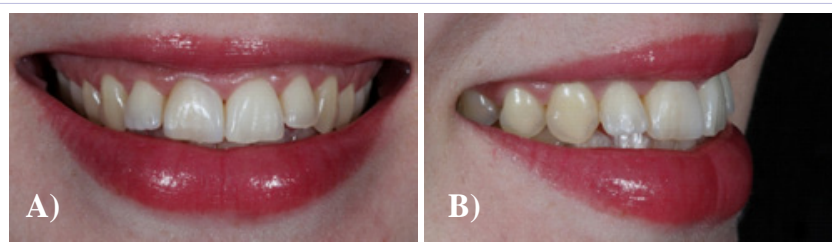

Figure 14: Patient's smile after one week A) Front view B) Lateral view.
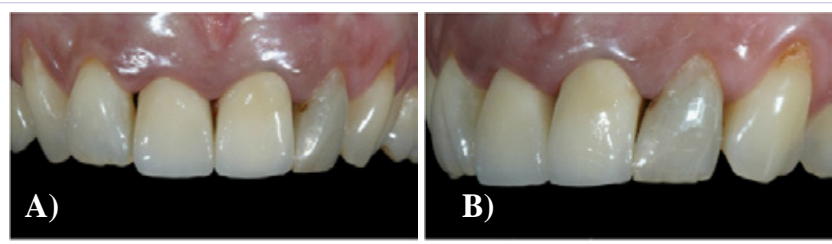

Figure 15: Right lateral and central incisor A) Frontal B) Lateral view.
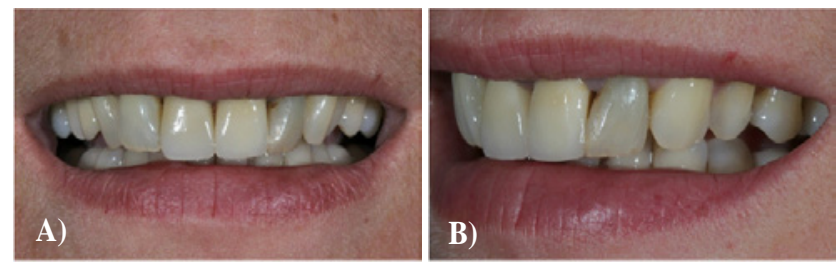

Figure 16: Initial smile of the patient A) Frontal B) Lateral view.

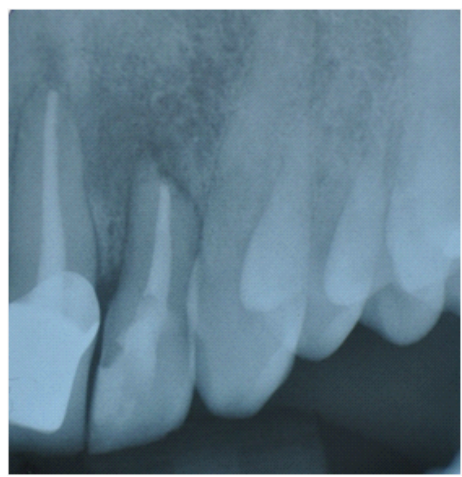

Figure 17: X-ray of the lateral treated incisor.

(Filtek Supreme Ultra, 3M ESPE AG, Seefeld, Germany).

Tooth shade selection was performed before field isolation to avoid color mismatches due to teeth' dehydration (Figure18A,B). A sagital silicone index was fabricated based on the diagnostic wax-up, in order to aid the correct tooth preparation. The upper front teeth were isolated with a medium weight rubber dam (Nictone, MDC Dental, Mexico) and dental floss was used for further stabilization of the rubber-dam. The preparation of the facial surface was 0.5-0.7 mm using a long, fine diamond bur with a chamfer margin under water-cooling, following the natural contours of the tooth (Figure 19A).

A $37.5 \%$ phosphoric acid (Gel-Etchant, Kerr Corporation, Orange, CA, USA) was applied to the prepared enamel for 30 seconds, rinsed with air-water spray for the same amount of time 
and air-dried. Adhesive procedures were performed with the application of a three-step etch-and-rinse system (Optibond FL, Kerr Corporation, Orange, CA, USA), according to manufacturer's instructions of use.

A portion of resinous opaque (Pink Opaque, Cosmedent, Chicago, IL, USA) captured at the tip of a flat painter's brush (Da Vinci, Series 374, Flat No. 4), was spread equally in a very thin layer on the discolored tooth surface and photopolymerized with a LED curing device (TechnoGaz, Parma, Italy) for 40 sec. The selection of the resinous Pink Opaquer was based on its potent but yet "warm" opacification result.
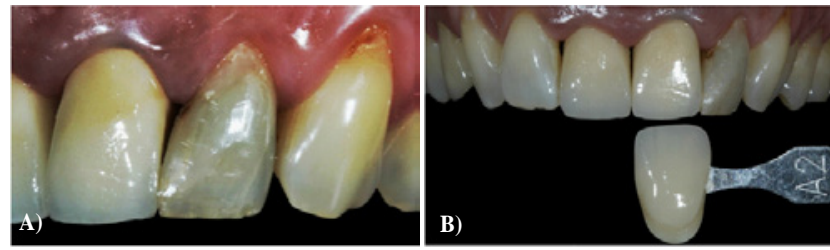

Figure 18: A) Digital processed contrast and brightness in order to highlight chromatic characteristics of the left lateral incisor B) Shade selection according to Vita Classical shade guide.

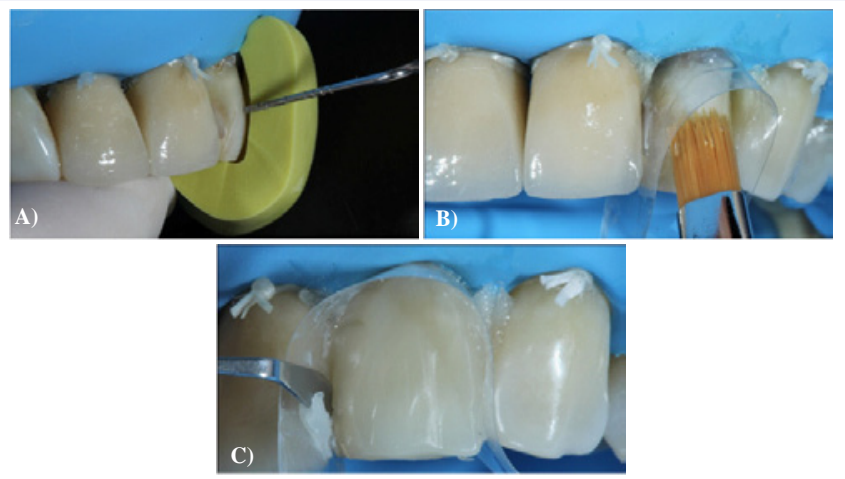

Figure 19: A) Check of the depth of the preparation with a silicone key B) Cervical application of the tint (Ice Effect Shade) on the polymerized dentin layer with a thin resin instrument C) Final layering and contouring of the enamel shade.
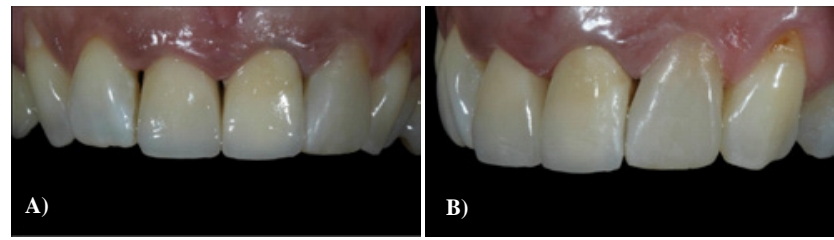

Figure 20: The final restoration A) Frontal B) Lateral view.
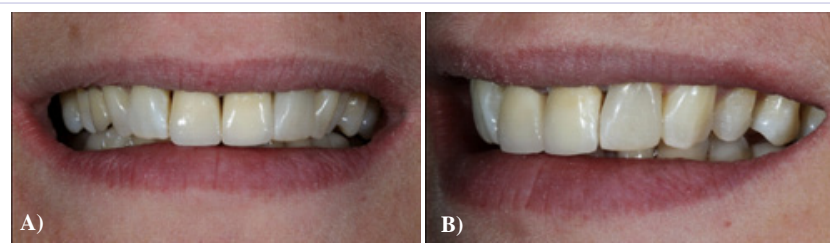

Figure 21: Final smile A) Frontal B) Lateral view.
Then a 0.3-0.5 mm increment of body, shade A2, resin composite (Filtek Supreme Ultra, 3M ESPE AG) was stratified and shade modifiers were used on the cervical part (Ice and Chroma Effect Shades, Inspiro, Edelweiss DR AG, Mercandor, Switzerland) in order to diminish the cervical value of the chosen shade (Figure19B). Finally, the enamel layer was reproduced with approximately $0.2 \mathrm{~mm}$ of A1 Enamel shade (Filtek Supreme Ultra, 3M ESPE AG), (Figure 19C).

Extra-fine diamond burs and fine aluminum oxide disks (SofLex, 3M ESPE AG, Seefeld, Germany) were used for finishing, prior to polishing with a two-step silicon-rubber polishing system (FlexiCups, Cosmedent, Chicago, IL, USA). Finally, a $5 \mu$ m diamond polishing paste (Diamond Polish Mint, Ultradent Products Inc, South Jordan) was used, in order to achieve a higher surface gloss. The final restorations (Figure 20A,B) and the overall smile were photo-documented after seven days (Figure 21A,B).

\section{Discussion}

The conservative handling of a single discolored incisor in the aesthetic zone can be a demanding task for the restorative dentist. Intrinsic discoloration in this area can be confronted with several treatment plans. Home or in-office bleaching for vital teeth and internal bleaching for non-vital teeth, is considered as the least invasive approach as it does not involve any facial tooth preparation [13]. However, in cases of endodontically treated teeth, careful evaluation should be conducted concerning the success of the endodontic therapy, prior to the initiation of the internal bleaching procedure. Internal bleaching is also contraindicated in the presence of a discolored tooth with cervical resorption [13]. The above can only prove the importance of thorough dental history investigation during treatment's planning of a discolored tooth in the aesthetic zone.

If a bleaching procedure cannot be implemented or has been tried out without a pleasing outcome and subsequent color rebounds, other conservative procedures should be considered, such as the placement of direct or indirect veneers. Ceramic materials are known for retaining their luster through time and accumulating lower amounts of plaque when compared to restorative materials like composites, especially when not properly polished [14-16]. Direct composite veneers on the other hand, can be completed in a single dental appointment; offer ease of reparability, are more cost-effective and often require minimal or no tooth preparation [14].

The longevity of direct composite veneers has not yet been thoroughly investigated, when compared to the amount of data concerning the ceramic veneers. Nonetheless, a survival rate of over $80 \%$ can be acquired by different composite systems for almost three and a half years of in vivo veneer performance [17].

Finally, it should be mentioned that maintaining aesthetics and functionality of the direct composite veneers, is a matter that involves not only the clinician but the patient as well. Therefore, the clinician should be accustomed with the necessary steps of composite veneers' maintenance during recall appointments. More specifically he should limit the ultrasonic scaler use on 
the veneer boarders, use polishing pastes only at the presence of stains and be familiar with the repair composite protocol and materials. He should also inform the patient on the posttreatment instructions, in order to prolong the longevity of the composite veneers. First of all, the patient should get accustomed to brushing soflty and flossing twice a day. He should use alcoholfree mouthrinses and products with NaF only - when in need of fluoride. He should additionally avoid certain foods so as to minimize the veneer fracture risk and attend the dental recall appointments for repair of possible minor defects. Generally, the patient should realize that the longevity of the composite veneers in his mouth depends also on his overall oral hygiene and masticatory habits.

Finally, for augmenting the longevity of such restorations and avoid micro and major chipping or fractures from occuring it should be noted that especially in cases of maloclussion, precautions should be taken by adjusting the occlusion and using protective mouthtrays.

\section{Conclusions}

Restoring a single discolored tooth in the anterior region with composite resin and achieving ideal aesthetics is a demanding task and success cannot be guaranteed. The use of opaquers can ameliorate the dark tooth substrate effectively, as long as they are handled carefully, and providing that enough space for the subsequent composite stratification is present. The application of the necessary amount of materials, the meticulous layering, finishing and polishing of the composite resin veneer are factors of utmost importance for clinical success.

\section{About The Authors}

The clinical work described in this paper has been carried out by the authors. Furthermore they declare no financial interest whatsoever in dental products or companies mentioned in this article.

\section{References}

1. Sensi LG, Webley W. A simplified approach for layering composite resin restorations. Gen Dent. 2007;55(7):638-645.

2. Watts A, Addy M. Tooth discoloration and staining: a review of the literature. Br Dent J. 2001;190(6): 309-316.

3. Krastl G, Allgayer N, Lenherr P, Filippi A, Taneja P, Weiger R. Tooth discoloration induced by endodontic materials: a literature review. Dent Traumatol. 2013;29(1):2-7. doi:10.1111/j.16009657.2012.01141.x.
4. Meyenberg K. The ideal restoration of endodontically treated teeth -structural and esthetic considerations: a review of the literature and clinical guidelines for the restorative clinician. Eur J Esthet Dent 2013;8(2):238-268.

5. Baratieri LN, Araujo E, Monteiro S Jr. Color in natural teeth and direct resin composite restorations: essential aspects. Eur J Esthet Dent. 2007;2(2)172-186.

6. Joiner A. Tooth colour: a review of the literature. J Dent. 2004;32(1):312.

7. Ikeda T, Murata Y, Sano H. Translucency of opaque-shade resin composites. Am J Dent. 2004;17:127-130.

8. Kim SJ, Son HH, Cho BH, Lee IB, Um CM. Translucency and masking ability of various opaque-shade composite resins. J Dent. 2009;37:102107. doi:10.1016/j.jdent.2008.10.002.

9. An JS, Son HH, Qadeer S, Ju SW, Ahn JS. The influence of a continuous increase in thickness of opaque-shade composite resin on masking ability and translucency. Acta Odontol Scand. 2013;71(1):120-129. doi: 10.3109/00016357.2011.654250.

10. Felippe LA, Baratieri LN. Direct resin composite veneers: masking the dark prepared enamel surface. Quintessence Int. 2000;31(8):557-562.

11. Abeer Al-Khreisat. Evaluating the relative optical translucency of opaquers composite resins. JRMS. 2006;13(2):62-66.

12. Felippe LA, Monteiro S Jr, Baratieri LN, Caldeira de Andrada MA, Ritter AV. Using opaquers under direct composite resin veneers: an illustrated review of the technique. J Esthet Restor Dent. 2003;15(6):327-336.

13. Alqahtani MQ. Tooth-bleaching procedures and their controversial effects: A literature review. Saudi Dent J. 2014;26(2):33-46. doi:10.1016/j.sdentj.2014.02.002.

14. Gresnigt MM, Kalk W, Ozcan M. Randomized clinical trial of indirect resin composite and ceramic veneers: up to 3-year follow-up. J Adhes Dent. 2013;15(2):181-190.

15. Gawriołek M, Sikorska E, Ferreira LF, Costa AI, Khmelinskii I, Krawczyk A, et.al . Color and luminescence stability of selected dental materials in vitro. J Prosthodont. 2012;21(2):112-122.

16. Heydecke G, Zhang F, Razzoog ME. In vitro color stability of doublelayer veneers after accelerated aging. J Prosthet Dent. 2001;85(6):551557.

17. Gresnigt M, Kalk W, Özcan M. Randomized controlled split-mouth clinical trial of direct laminate veneers with two micro-hybrid resin composites. J Dent. 2012;40(9):766-775. 